\title{
A CCD imaging search for wide metal-poor binaries ${ }^{\star \star \star}$
}

\author{
M. R. Zapatero Osorio ${ }^{1}$ and E. L. Martín ${ }^{2}$
}

\author{
1 LAEFF - INTA, PO Box 50727, 28080 Madrid, Spain \\ 2 Instituto de Astrofísica de Canarias, 38200 La Laguna, Tenerife, Spain
}

Received 19 December 2003 / Accepted 12 February 2004

\begin{abstract}
We explored the regions within a radius of $25^{\prime \prime}$ around 473 nearby, low-metallicity G- to M-type stars using (VR)I optical filters and small-aperture telescopes. About $10 \%$ of the sample was searched up to angular separations of $90^{\prime \prime}$. We applied photometric and astrometric techniques to detect true physical companions to the targets. The great majority of the sample stars was drawn from the Carney-Latham surveys; their metallicities range from roughly solar to $[\mathrm{Fe} / \mathrm{H}]=-3.5 \mathrm{dex}$. Our I-band photometric survey detected objects that are between 0 and 5 mag fainter (completeness) than the target stars; the maximum dynamical range of our exploration is $9 \mathrm{mag}$. We also investigated the literature, and inspected images from the Digitized Sky Surveys to complete our search. By combining photometric and proper motion measurements, we retrieved 29 previously known companions, and identified 13 new proper motion companions. Near-infrared 2MASS photometry is provided for the great majority of them. Low-resolution optical spectroscopy (386-1000 nm) was obtained for eight of the new companion stars. These spectroscopic data confirm them as cool, late-type, metal-depleted dwarfs, with spectral classes from esdK7 to sdM3. After comparison with low-metallicity evolutionary models, we estimate the masses of the proper motion companion stars to be in the range $0.5-0.1 M_{\odot}$. They are moving around their primary stars at projected separations between $\sim 32$ and $\sim 57000 \mathrm{AU}$. These orbital sizes are very similar to those of solar-metallicity stars of the same spectral types. Our results indicate that about $15 \%$ of the metal-poor stars have stellar companions in wide orbits, which is in agreement with the binary fraction observed among main sequence G- to M-type stars and T Tauri stars.
\end{abstract}

Key words. stars: subdwarfs - stars: binaries: visual - stars: statistics

\section{Introduction}

Halo subdwarfs are metal-deficient $([\mathrm{Fe} / \mathrm{H}] \leq-1 \mathrm{dex})$, highvelocity $\left(v_{\tan } \geq 200 \mathrm{~km} \mathrm{~s}^{-1}\right)$ stars. They belong to the oldest known galactic population and are subluminous with respect to main-sequence stars of the same mass. Traditionally, they have been identified using proper motion surveys (e.g., Giclas 1971) and, more recently, objective prism plates (e.g., Beers et al. 1985). Such surveys are magnitude-limited and consequently biased towards intrinsically brighter stars. Only recently has a subdwarf sequence of very-low mass halo

Send offprint requests to: M. R. Zapatero Osorio,

e-mail: mosorio@laeff.esa.es

* Based on observations made with the IAC80 telescope operated on the island of Tenerife by the Instituto de Astrofísica de Canarias in the Spanish Observatorio del Teide; also based on observations made with the $2.2 \mathrm{~m}$ telescope of the German-Spanish Calar Alto Observatory (Almería, Spain), the William Herschel Telescope (WHT) operated on the island of La Palma by the Isaac Newton Group in the Spanish Observatorio del Roque de los Muchachos (ORM) of the Instituto de Astrofísica de Canarias; and the Telescopio Nazionale Galileo (TNG) at the ORM.

$\star \star$ The complete Table 1 is only available in electronic form at the CDS via anonymous ftp to

cdsarc.u-strasbg.fr $(130.79 .128 .5)$ or via

http://cdsweb.u-strasbg.fr/cgi-bin/qcat?J/A+A/419/167 subdwarfs been identified, with a cutoff at $M(V)=14.5$ and $(V-I)=2.8 \mathrm{mag}$ (Monet et al. 1992). The bolometric luminosities, effective temperatures and metallicities of these stars, and the faint cutoff, are an important constraint to stellar structural and evolutionary models. Gizis \& Reid (1997) have stressed the importance of wide binary systems for checking the metallicity scale of low-mass dwarfs.

In 1991 we started a CCD-based imaging survey aimed at finding wide low-mass companions to halo subdwarfs. Wide binary systems are like small clusters because they offer us the possibility of studying two stars of different mass, but with the same age, distance and chemical composition. Early results of our survey were reported in Martín \& Rebolo (1992) and Martín et al. (1995). In this paper, we present the full survey, including the discovery of companions to 13 metaldeficient dwarfs. Spectroscopic observations have been obtained for eight of the low-mass secondaries. We derive spectral types for these new objects ranging from esdK7 to sdM3.

\section{Sample selection}

We selected 473 G-, K- and M-type stars with known lowmetallicities for our astrometric/photometric search. They were drawn from the surveys by Hartwick et al. (1984), Laird et al. (1988), Schuster \& Nissen (1989) and 
Table 1. List of photometric and astrometric observations.

\begin{tabular}{lcccrccrrrr}
\hline \hline Object & $V_{\mathrm{p}}$ & Fil. & Tel. & $\begin{array}{r}\text { Exp. } \\
(\mathrm{s})\end{array}$ & $\begin{array}{r}F W H M \\
\left({ }^{\prime \prime}\right)\end{array}$ & Date & $\begin{array}{r}\rho \\
\left({ }^{\prime \prime}\right)\end{array}$ & $\begin{array}{r}\text { PA } \\
(\mathrm{deg})\end{array}$ & $\Delta$ mag & Notes \\
\hline G001-004 & 10.69 & $I$ & IAC80 & 35 & 1.9 & $01 / 08 / 94$ & $\geq 25$ & & & \\
G001-012 & 13.31 & $I$ & IAC80 & 100 & 2.6 & $02 / 08 / 94$ & 23.29 & 276.8 & 4.49 & 1 \\
G001-013 & 15.23 & $I$ & IAC80 & 330 & 2.5 & $02 / 08 / 94$ & 20.48 & 341.2 & 3.89 & 1 \\
\hline
\end{tabular}

Notes: this is an example of the table. The complete table is available in electronic form at the CDS.

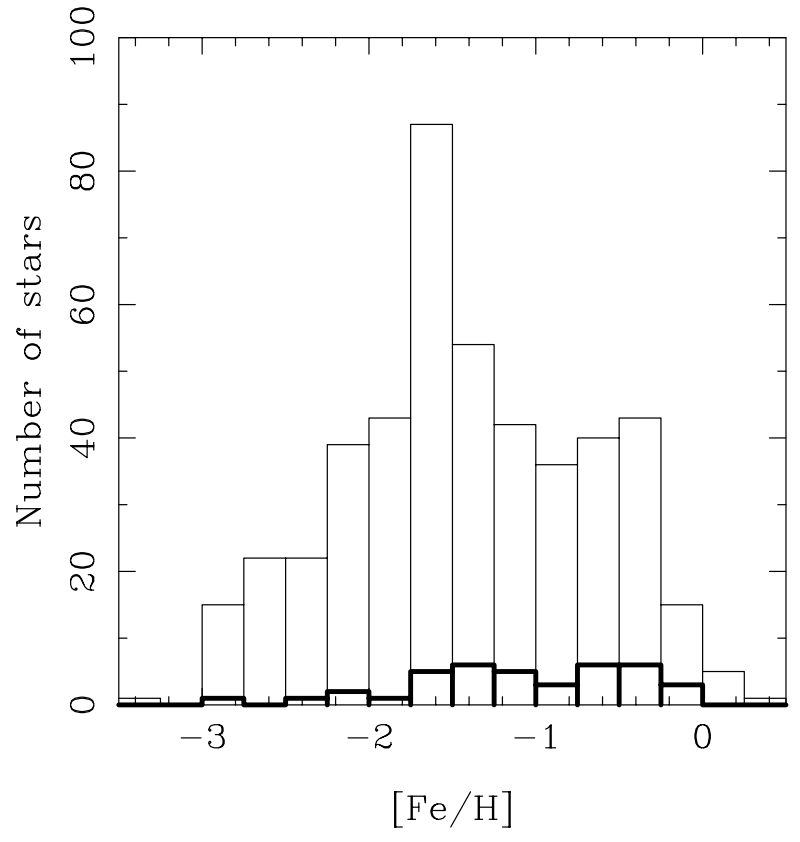

Fig. 1. The metallicity distribution of our entire sample is plotted as a thin line. The metallicity distribution of the stars with wide companions is displayed with a thick line. The bin width represents a change of 0.25 dex in the metal logarithmic abundance.

Carney et al. (1994). Chemical composition, as inferred from the $[\mathrm{Fe} / \mathrm{H}]$ measurements given by the previous references, Ryan \& Norris (1991) and Cayrel de Strobel et al. (1992), is between solar abundance $(0.0 \mathrm{dex})$ and $-3.5 \mathrm{dex}$. The method of Carney et al. (1994), which is the source of the great majority of our targets, to derive metal abundances is the best fit between observed spectra and a grid of synthetic spectra at the same effective temperatures but with varying metallicites. We will refer to these metallicity estimates as $[\mathrm{Fe} / \mathrm{H}]$ throughout this paper. The distribution of metallicities is shown in Fig. 1: about $50 \%$ of the sample has a metal abundance between one and two orders of magnitude more depleted than the Sun, $\sim 30 \%$ of the stars have metallicities between 0.0 and $-1.0 \mathrm{dex}$, and the remaining $\sim 20 \%$ of the stars are metal-depleted by more than a factor of 100 .

Additionally, the sample comprises high proper motion stars (typically $\mu \geq 0.1^{\prime \prime} / y r$ ), and with few exceptions, optical magnitudes are brighter than $14.5 \mathrm{mag}$. The distance to the targets is between 15 and 1000 pc in all cases, except for one Hipparcos star, as shown in Fig. 2. About $46 \%$ of the sample stars have Hipparcos parallaxes (Perryman et al. 1997).

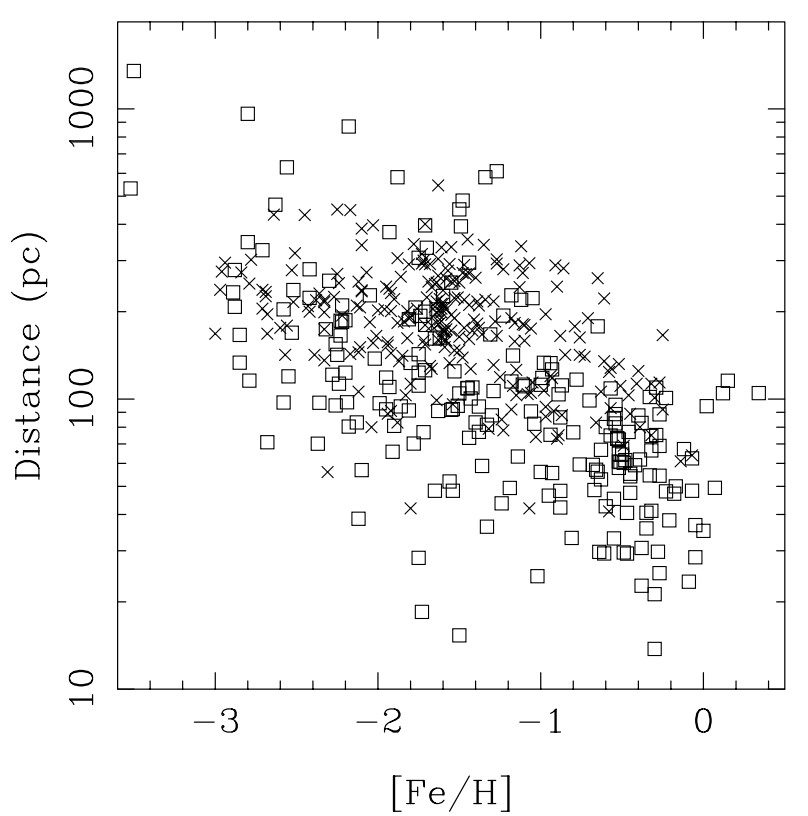

Fig. 2. Distance and metallicity of our target stars. Hipparcos stars (46\% of the sample) are plotted as open squares, while crosses stand for stars with distances from Carney et al. (1994).

Interestingly, Fig. 2 also depicts the relation between metallicity and distance for nearby stars: in general terms, the most metal-depleted stars are located farther away than the most metal-rich stars. The lower envelope to this relation is given by $\log d(\mathrm{pc})=1.146-0.268[\mathrm{Fe} / \mathrm{H}]$. We used only Hipparcos data to derive this equation.

The list of all of our targets appears in Table 1. Stars are listed in order of increasing Giclas designation (Giclas 1971). Those stars with no Giclas name are listed at the end of the table. We also provide $V$ magnitudes in the second column. We remark that we did not apply any particular criteria for the object selection, except for the metal-poor nature of the stars.

\section{Observations and analysis}

\subsection{Optical photometric and astrometric survey}

The great majority of the direct imaging observations was conducted with the $0.8 \mathrm{~m}$ IAC 80 telescope at the Observatorio del Teide during 1994, 1995, 1996, 2002, and 2003. The telescope was equipped with a Thomson $1024 \times 1024 \mathrm{CCD}$ camera, which provided a pixel projection onto the sky of 0 ' 4325 . Further optical observations were carried out with the $1024 \times 1024 \mathrm{CCD}$ 
cameras of the $1 \mathrm{~m}$ Jacobus Kapteyn Telescope (JKT) and of the $2.5 \mathrm{~m}$ Nordic Optical Telescope (NOT) at the Observatorio del Roque de los Muchachos (ORM) during 1991, 1993, and 1994. The corresponding plate scales were 0!30 (JKT) and 0 '. 14 (NOT). The most recent images taken in 2003 were obtained with the IAC80 telescope (same instrumental configuration as in the past) and the CAFOS instrument attached to the $2.2 \mathrm{~m}$ telescope at the Calar Alto Observatory (CAHA), providing a pixel size of 0. '53. Some observing nights were hampered by cirrus. Hence, we did not perform any absolute photometric calibration. The survey was entirely conducted in the $I$ filter because faint dwarf companions are significantly redder than their primary stars. Some targets were also imaged in the $V$ and/or $R$ filters to determine color differences between targets and companion candidates. This program was usually carried out as a backup observing program whenever the seeing was rather poor $\left(\geq 2^{\prime \prime}\right)$ or there were many clouds.

Raw frames were bias-subtracted and flat-field corrected using packages running inside the $\operatorname{IRAF}^{1}$ environment. Flatfields were usually collected during dawn and dusk. Individual exposures ranged between a few seconds and $600 \mathrm{~s}$, depending on the brightness of the targets and the telescope diameter. These integration times did not yield deep images. However, these data are intended to detect objects typically between 0 and 5 mag fainter than the target stars. This is the dynamical magnitude range for which our survey is complete. In some cases, less luminous objects are seen (maximum dynamical range is $9 \mathrm{mag}$ ). Observing dates, telescope, filters, exposure times, and the mean spatial resolution calculated as the average FWHM of the images are all given in Table 1.

We performed differential photometry and relative astrometry of all star-like sources that are found within a radius of $25^{\prime \prime}$ from the low-metallicity source. For about $10 \%$ of our sample, we also investigated up to 1'5. Based on the lower envelope delineated by the distance-metallicity relation of Fig. 2, and for an average seeing of 2" (see Table 1), we can resolve projected separations larger than $30 \mathrm{AU}$ for roughly solar metallicities, and larger than $200 \mathrm{AU}$ for $[\mathrm{Fe} / \mathrm{H}]=-3$. Instrumental magnitudes of both targets and companion candidates are determined by considering constant circular apertures that do not overlap with other sources. The magnitude difference of a candidate with respect to its metal-depleted star is computed by subtracting instrumental magnitudes. For bright sources, photometry is accurate to $\pm 0.05 \mathrm{mag}$. The photometric uncertainty increases up to $\pm 0.25 \mathrm{mag}$ for the faintest sources. Photometric diagrams will be discussed in Sect. 4.

Projected separations and position angles are calculated from the centroids of the objects, which are obtained by fitting a Gaussian function to the radial profile distribution of the sources. Common proper motion visual binaries are recognized because the members of a system should keep a rather constant separation and position angle within intervals of years, while changing their apparent location with respect to background

${ }^{1}$ IRAF is distributed by National Optical Astronomy Observatory, which is operated by the Association of Universities for Research in Astronomy, Inc., under contract with the National Science Foundation. sources. We note that the accuracy of the centroids of bright sources is typically \pm 0.2 pix, and being about \pm 1 pix for objects close to the detection limit. This, in addition to the uncertainty of the CCD plate scales, yield astrometric precisions between $0{ }^{\prime} 05$ and $0{ }^{\prime} 4$. The uncertainty in position angles is in the range $0.5-1 \mathrm{deg}$, which also accounts for small offsets in the N-E orientation of the instruments. We summarize our photometric and astrometric results in Table 1.

Target stars with nearby red companion candidates were observed at different epochs years apart, allowing us to identify common proper motion pairs. We also used red images provided by the Digitized Sky Surveys to complete our astrometric study. In addition, we searched the literature and SIMBAD for identifying wide binaries with large or with very small separations, which we cannot resolve in our data. As a result, we found that G 128-064 and G 153067 have proper motion companions located at 4!3 (Allen et al. 2000) and 16!6 (Gliese \& Jahreiss 1988), respectively, which obviously are off limits of the detectors used in our survey. We also found Hipparcos binaries (e.g., G230-049, G273-001) that have separations significantly smaller than the typical spatial resolution of our images.

Of the 473 stars in the original sample, we detected 29 previously known binaries and multiple systems with separations between 0'.17 and 17' (Luyten 1979; Perryman et al. 1997; Salim \& Gould 2003), and 13 new proper motion companions with separations between $2^{\prime \prime}$ and 1'9. Their photometric and astrometric data are listed in Tables 2 (known pairs) and 3 (new pairs). In these tables, we provide the name of the companion, the metallicity and optical magnitudes of the primary star, the Hipparcos distance to the system (when available), the distance given by Carney et al. (1994), and the projected separation in AU (the Hipparcos distance is preferred over Carney et al.'s value). Optical magnitudes of primaries were taken from the Hipparcos database as well as from the literature (Stauffer \& Hartmann 1986; Ryan 1989; Greenstein 1989; Weis 1991; Ryan 1992).

Three of the new proper motion companions in Table 3 (G 090-036 B, G 116-009 B, and G 176-046 D) were previously reported by Martín et al. (1995). They are early discoveries from the survey described here. The optical low-resolution spectroscopy of G 116-009 B and G 176-046D, presented in this paper and in Gizis \& Reid (1997) confirm them as lowmetallicity late-type subdwarfs.

\section{2. $2 M A S S$ near-infrared photometry}

Table 4 provides the $J H K_{\mathrm{s}}$ magnitudes of both the primary and secondary stars, which we obtained from the 2MASS all-sky survey data archive. All the sources are unambigously identified. From our list, companions separated by less than 4" are not well spatially resolved by 2MASS. In these systems, except for G 204-049, photometry is contaminated by the presence of the companion (see the notes to the table). We remark that the 2MASS $J$ and $H$ magnitudes of G 176-046 A and G 176-046D are only upper limits to the real values; in contrast, the $K_{\mathrm{s}}$ data 
Table 2. Previously known proper motion companions.

\begin{tabular}{|c|c|c|c|c|c|c|c|c|c|c|c|c|c|}
\hline Companion & Primary & {$[\mathrm{Fe} / \mathrm{H}]$} & $V_{\mathrm{p}}$ & $R_{\mathrm{p}}$ & $I_{\mathrm{p}}$ & $\Delta(V)$ & $\Delta(R)$ & $\Delta(I)$ & $\begin{array}{c}\rho \\
\left({ }^{\prime \prime}\right)\end{array}$ & $\begin{array}{c}\text { PA } \\
(\operatorname{deg})\end{array}$ & $\begin{array}{l}\text { Sep. } \\
\text { (AU) }\end{array}$ & $\begin{array}{r}d \text { (Hip) } \\
(\mathrm{pc})\end{array}$ & $\begin{array}{r}d^{a} \\
(\mathrm{pc})\end{array}$ \\
\hline G 009-003 B ${ }^{b}$ & G 009-003 A & & 13.07 & & & 5.92 & 5.25 & 4.15 & 15.88 & 63.0 & & & \\
\hline G 026-008 B & G 026-008 A & -0.32 & 10.47 & & & 4.70 & 4.16 & 3.26 & 8.48 & 306.0 & 636 & & 75 \\
\hline $\mathrm{G} 026-010^{c}$ & G 026-009 & -0.99 & 9.89 & & & 5.04 & 5.53 & 6.19 & 133.04 & 29.3 & 6572 & 49.4 & \\
\hline G 027-008 B & G 027-008 A & -1.53 & 11.39 & 11.06 & 10.70 & 5.71 & 5.13 & 4.62 & 5.63 & 173.3 & 828 & & 147 \\
\hline LP 581-80 & G 028-040 & -0.87 & 11.33 & & 8.17 & 3.20 & & 2.25 & 15.81 & 349.8 & 1873 & 111.5 & 114 \\
\hline $\mathrm{G} 032-046 \mathrm{~B}^{d}$ & G 032-046 A & -0.57 & 9.79 & & 9.02 & 8.81 & & 8.74 & 62.52 & 168.1 & 4679 & 74.9 & 59 \\
\hline $\mathrm{G} 061-023^{e}$ & G 061-024 & -0.53 & 8.99 & & & 0.77 & & & 81.90 & 221.7 & 5987 & 73.1 & 69 \\
\hline GJ 516 B & G 063-036 $6^{f}$ & & 11.38 & 9.84 & 8.84 & 0.3: & & 0.3 : & 2.7: & 40: & 37 & 13.7 & \\
\hline $\mathrm{BD}+062932 \mathrm{~B}$ & G 066-022 & -1.30 & 10.47 & 10.09 & 9.67 & 3.19 & & 2.24 & 3.60 & 250.5 & 316 & 87.9 & 61 \\
\hline G 069-004 $\mathrm{N}^{g}$ & G 069-004 & -0.63 & 7.98 & & 7.22 & 0.84 & 0.87 & 0.81 & 6.16 & 22.9 & 325 & 52.8 & 31 \\
\hline G 095-057 B ${ }^{h}$ & G 095-057 A & -1.02 & 8.15 & 8.22 & 7.32 & 0.61 & 0.53 & 0.44 & 7.25 & 54.3 & 178 & 24.4 & 22 \\
\hline G $128-064 \mathrm{~B}^{i}$ & G 128-064 A & -0.27 & 9.63 & & 8.80 & 2.10 & & 2.13 & 261.60 & 320.3 & 23177 & 88.6 & 65 \\
\hline $\mathrm{G} 017-027^{j}$ & G 153-067 & -1.65 & 9.63 & 9.14 & 8.70 & 4.25 & & & 1170.70 & 36.7 & 56544 & 48.3 & 35 \\
\hline G 171-050 B & G 171-050 A & -2.94 & 13.08 & & & 5.25 & 4.72 & 4.32 & 22.48 & 268.4 & 6632 & & 295 \\
\hline G 173-003 & G 173-002 & -0.07 & 10.05 & & & 4.16 & 3.63 & 2.82 & 22.51 & 34.6 & 1440 & & 64 \\
\hline G 194-037 B & G 194-037 A & -2.03 & 15.31 & & & 2.63 & 2.53 & 1.86 & 19.71 & 48.2 & 7825 & & 397 \\
\hline G $197-050^{k}$ & G 197-049 & -1.50 & 9.86 & 8.86 & 8.08 & 3.72 & 3.08 & 2.71 & 14.68 & 9.4 & 225 & 15.3 & \\
\hline G 217-004 B & G 217-004 A & -0.40 & 11.46 & & 10.64 & 6.85 & 6.06 & 4.98 & 12.36 & 200.8 & 1085 & 87.8 & 137 \\
\hline G 230-047 B ${ }^{l}$ & G 230-047 A & -0.39 & 10.12 & & & 5.01 & 4.49 & 3.63 & 11.63 & 267.3 & 930 & & 80 \\
\hline G 230-049 B ${ }^{m}$ & G 230-049 A & -0.66 & 8.51 & & 7.89 & 0.11 & & & 1.33 & 29.0 & 76 & 57.1 & 43 \\
\hline G 242-074 $\mathrm{B}^{n}$ & G 242-074 A & -0.78 & 9.78 & & 9.09 & 5.1: & & 4.08 & 19.50 & 336.1 & 2276 & 116.6 & 91 \\
\hline $\mathrm{G} 251-054 \mathrm{~B}^{o}$ & G 251-054 A & -2.36 & 10.07 & & 9.37 & 4.60 & 4.25 & 4.05 & 110.59 & 208.5 & 28288 & 255.8 & 59 \\
\hline $\mathrm{G} 252-049 \mathrm{~B}^{p}$ & G 252-049 A & $\geq-0.3$ & 9.48 & & 7.93 & 0.11 & & & 1.53 & 112.0 & 32 & 21.2 & \\
\hline G $255-038 \mathrm{~B}^{q}$ & G 255-038 A & & 9.78 & 9.12 & 8.57 & 3.78 & & 2.36 & 14.34 & 324.2 & 644 & 44.9 & \\
\hline G 262-022 $2^{r}$ & G 262-021 & -1.07 & 13.75 & 13.40 & 12.96 & 0.68 & 0.42 & 0.19 & 29.54 & 148.4 & 8685 & & 294 \\
\hline G 266-029 B ${ }^{s}$ & G 266-029 A & -1.18 & 8.96 & & 8.61 & 0.40 & & & 0.17 & & 39 & 227.8 & \\
\hline LHS 541 & G 273-001 ${ }^{t}$ & -1.40 & 8.16 & 7.87 & 7.54 & 8.16 & 7.60 & 6.67 & 18.57 & 52.0 & 1542 & 83.0 & 37 \\
\hline $\mathrm{BD}+162116 \mathrm{~B}^{u}$ & $\mathrm{BD}+162116 \mathrm{~A}$ & -0.05 & 7.48 & & 6.77 & 1.8: & & & 1.2: & $256:$ & 34 & 28.5 & \\
\hline $\mathrm{BD}+232207 \mathrm{~B}^{v}$ & $\mathrm{BD}+232207 \mathrm{~A}$ & -0.38 & 5.80 & & 5.23 & 5.8: & & 4.33 & 7.72 & 302.7 & 175 & 22.7 & \\
\hline
\end{tabular}

${ }^{a}$ Distance from Carney et al. (1994), except for G 176-046 D.

${ }^{b} V$ magnitude from Salim et al. (2003).

${ }^{c}$ White dwarf. Metallicity from Cayrel de Strobel et al. (1997).

${ }^{d}$ CCDM J00453+1421 B.

${ }^{e} \Delta V$ and astrometry from Salim et al. (2003). $I$ (G 061-023) = 9.03 mag (Hipparcos).

${ }^{f}$ G 063-036 is a low-metallicity, M-type star (Stauffer \& Hartmann 1986).

${ }^{g}$ Hipparcos separation and position angle are 6.' 193 and 23 deg, respectively.

${ }^{h}$ Hipparcos separation and position angle are 7"'307 and 54 deg, respectively.

${ }^{i} \mathrm{G} 128-064 \mathrm{~B}$ is possibly HIP 115671. $\Delta V$ and astrometry from Salim et al. (2003). $\Delta I$ from Hipparcos.

${ }^{j} \Delta V$ from SIMBAD. Astrometry from from Salim et al. (2003). G 153-067 is also known as G 017-025.

${ }^{k} \Delta R$ from Weis (1991). Metallicity from Alonso et al. (1996).

${ }^{l}$ CCDM J20415+5730 B.

${ }^{m}$ Not resolved in our images. Separation, position angle and $\Delta V$ from the Hipparcos database.

${ }^{n}$ CCDM J00582+8007 B.

${ }^{\circ}$ CCDM J08111+7954 B.

${ }^{p}$ Not resolved in our images. Astrometry and $\Delta V$ from Hipparcos; metallicity from Reid et al. (2001).

${ }^{q}$ CCDM J13349+7430 B. Hipparcos separation and position angle are 14"' 28 and 324 deg, respectively. See notes to Table 1.

${ }^{r} \Delta V$ from SIMBAD.

${ }^{s}$ Not resolved in our data. Astrometry and $\Delta V$ from Allen et al. (2000).

${ }^{t}$ G 273-001 is an Hipparcos binary (see text and notes to Table 1).

" Hipparcos separation of 1". 81 and PA = $246 \mathrm{deg}$. Metallicity from Zakhozhaj \& Shaparenko (1996).

${ }^{v}$ Metallicity from Cayrel de Strobel et al. (1992). 
Table 3. New proper motion companions.

\begin{tabular}{|c|c|c|c|c|c|c|c|c|c|c|c|c|}
\hline "Companion & 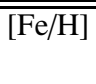 & $\overline{V_{\mathrm{p}}}$ & $\overline{R_{\mathrm{p}}}$ & $\overline{\overline{I_{\mathrm{p}}}}$ & 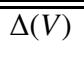 & $\overline{\overline{\Delta \Delta(R)}}$ & 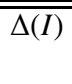 & $\begin{array}{c}\rho \\
\left({ }^{\prime \prime}\right)\end{array}$ & $\begin{array}{c}\text { PA } \\
\text { (deg) }\end{array}$ & $\begin{array}{l}\text { Sep. } \\
\text { (AU) }\end{array}$ & $\begin{array}{c}d \text { (Hip) } \\
(\mathrm{pc})\end{array}$ & $\begin{array}{c}d^{a} \\
(\mathrm{pc})\end{array}$ \\
\hline G 009-047 B ${ }^{b}$ & -1.93 & 7.70 & & 6.88 & 8.70 & 8.23 & 8.05 & 81.93 & 229.5 & 30800 & 375.94 & 13 \\
\hline $\mathrm{G} 059-032 \mathrm{~B}^{c}$ & -0.23 & 9.04 & & 8.10 & & & & 112.03 & 108.7 & 5381 & 48.05 & 58 \\
\hline G 090-036 B & -1.62 & 12.69 & & & 3.44 & 2.97 & 2.61 & 1.67 & 47.4 & 369 & & 221 \\
\hline $\mathrm{G} 093-027 \mathrm{~B}^{d}$ & -1.23 & 11.64 & & & 2.7: & 2.2: & 1.8: & 3.52 & 101.3 & 275 & & 78 \\
\hline G 116-009 B & -1.46 & 14.34 & & & 4.00 & 3.57 & 3.03 & 10.08 & 90.5 & 2712 & & 269 \\
\hline G 128-077 B & -1.36 & 13.36 & & & 2.87 & 2.05 & 1.89 & 9.18 & 138.3 & 1533 & & 167 \\
\hline G 172-016 B & -1.64 & 10.97 & & & 5.85 & 5.34 & 4.84 & 8.30 & 154.5 & 822 & & 99 \\
\hline $\mathrm{G} 176-046 \mathrm{D}^{b}$ & -1.67 & 12.59 & 12.16 & 11.70 & 4.89 & 4.76 & 3.89 & 4.80 & 152.9 & 610 & & 127 \\
\hline G 188-022 B & -1.45 & 10.05 & & 9.55 & 6.93 & 6.21 & 5.45 & 5.08 & 308.3 & 553 & 108.81 & 89 \\
\hline G 204-049 B & -0.97 & 10.85 & & & 4.3: & 3.7: & 3.1: & 2.7: & 72: & 235: & & 87 \\
\hline G 214-001 B & -2.03 & 12.08 & & & 5.74 & 4.95 & 4.59 & 5.14 & 353.0 & 802 & & 156 \\
\hline G 216-045 B & -0.52 & 11.24 & & & 8.56 & 7.49 & 6.22 & 31.45 & 43.9 & 4151 & & 132 \\
\hline G 273-152 B & -0.60 & 10.10 & 9.65 & 9.23 & $\leq 5.7$ & 4.42 & 3.57 & 3.84 & 277.6 & 307 & 80.06 & 65 \\
\hline
\end{tabular}

\footnotetext{
${ }^{a}$ Distance from Carney et al. (1994), except for G 009-047 B.

${ }^{b}$ Distance and metallicity from Laird et al. (1988).

${ }^{c}$ Astrometry from the Digital Sky Survey images.

${ }^{d}$ According to Carney et al. (1994) it is a visual binary, but nothing is found in either SIMBAD or the archives.
}

are reliable because the two objects were resolved by 2MASS and the photometry was obtained by a profile-fitting technique. Because of contaminated or unresolved near-infrared photometry, the following new pairs are not included in the near-infrared plots of Sect. 4: G 090-036, G 093-027, G 176-046, G 204-049, and G 273-152.

\subsection{Optical spectroscopy}

We obtained optical low-resolution spectroscopy of eight new proper motion companions listed in Table 5. Six of them were observed using the double-arm ISIS spectrograph and the $4.2 \mathrm{~m}$ William Herschel Telescope (WHT) at the ORM. For comparison purposes, we also observed BD +062932 B (companion to G 066-022) and LHS 541 (vB 12), the low-metallicity dwarf companion to LHS 540 (G273-1), which was discovered by van Biesbroeck (1961). On 2002 Aug. 23 we used the $570 \mathrm{~nm}$ dichroic, the R600R grism in the red arm, and the R300B grism in the blue arm. The pixel size of the EEV CCD detectors and the binning of a factor of 2 along the spectral direction in the red arm provided nominal dispersions of $0.865 \AA$ /pix (blue) and $0.889 \AA /$ pix (red). On 2003 Sep. 11 we only used the red arm, the R158R grism, the order-blocking filter GG495, and the same detector as in the previous run. This instrumental setup provided a lower nominal dispersion of $1.63 \AA$ /pix (no binning was applied). Older data were taken on 1994 Jul. 1-2, using the $\mathrm{R} 158 \mathrm{~B}$ grating in the blue arm and the $\mathrm{R} 158 \mathrm{R}$ grating in the red arm, which provided nominal dispersions of 2.9 and $2.7 \AA /$ pix, respectively. We always observed through a slit-width of $1{ }^{\prime \prime}$; all data were collected at parallactic angle to minimize light losses. The seeing in the visible was rather poor (2") on 23 Aug. 2002 and in Jul. 1994, and 1" on 11 Sep. 2003. The latter night was hampered by clouds and was clearly non-photometric.

Optical spectra of two slightly metal-poor new companions were obtained with the DOLORES spectrograph and the
$3.5 \mathrm{~m}$ Telescopio Nazionale Galileo (TNG) at the ORM on 2003 Dec. 12. We used a slit width of 1".5, the low-resolution grism LR-R and a $2048 \times 2048$ LORAL detector, which provided a nominal dispersion of $2.88 \AA /$ pix and a final resolution of $13 \AA$. These observations were not carried out at parallactic angle.

Raw frames were bias-subtracted and flat-field corrected using standard techniques. Spectra were optimally extracted with the TWODSPEC package of IRAF. The error in wavelength calibration, which was performed with atomic lines of $\mathrm{Cu}, \mathrm{Ar}$ and $\mathrm{Ne}$, is around $2.5-5 \%$. Instrumental response was removed to an accuracy of $10 \%$ using observations of the spectroscopic standard stars G 191-B2B, BD+25 3941, BD+28 4211 and Hilter 102, which have fluxes available in the IRAF database. The log of the spectroscopic observations appears in Table 5, where we include exposure time per object, final spectral resolution, and spectral coverage.

The resulting spectra are depicted in Figs. 3-5. The 2003 WHT and TNG spectra are affected by a strong fringing pattern redwards of $800 \mathrm{~nm}$. We did not attempt to remove telluric features from the WHT data because of the very fast changes of the sky conditions during the observations. In contrast, the TNG data were reasonably well corrected for telluric absorption and fringing, although some fringing residuals are observed in the spectrum of G 273-152 B (Fig. 4). The 1994 and 2002 red data were also corrected for telluric absorption. This was accomplished by observing G-type stars at similar airmass shortly before the target observation and interpolating the continuum across the telluric bands. This correction leads to a better detection of the K I atomic doublet at 766.5 and $769.9 \mathrm{~nm}$, as shown in the upper panel of Fig. 3.

The spectra show enhanced absorption features of the hydrides $\mathrm{MgH}$ and $\mathrm{CaH}$, labeled in Figs. 3 and 5, which are typical of cool, low-metallicity subdwarfs. Regarding oxides, the data have rather weak (as compared to solar-metallicity stars of 
Table 4. 2MASS photometry of primaries and companions.

\begin{tabular}{|c|c|c|c|c|c|c|c|}
\hline Object & $J$ & $H$ & $K_{\mathrm{s}}$ & Object & $J$ & $H$ & $K_{\mathrm{s}}$ \\
\hline G 009-003 A & $9.676(.021)$ & $9.069(.021)$ & $8.843(.022)$ & G 172-016 B & $13.992(.041)$ & $13.408(.049)$ & $13.195(.042)$ \\
\hline G 009-003 B & $3.182(.021)$ & $12.584(.027)$ & $12.321(.023)$ & G 173-002 & $8.492(.019)$ & $8.048(.018)$ & $7.976(.023)$ \\
\hline G 009-047 A & $6.105(.034)$ & $5.594(.027)$ & $5.551(.021)$ & G 173-003 & $10.752(.018)$ & $10.174(.015)$ & $9.903(.020)$ \\
\hline G 009-047 B & $14.051(.028)$ & $13.366(.036)$ & $13.191(.030)$ & $\mathrm{G} 176-046 \mathrm{~A}^{e}$ & 11.093( & 10.608( & $10.496(.026)$ \\
\hline G 026-008 A & $8.877(.023)$ & $8.440(.055)$ & $8.352(.021)$ & $\mathrm{G} 176-046 \mathrm{D}^{e}$ & $12.276(\quad)$ & $11.822(\quad)$ & $13.501(.049)$ \\
\hline G 026-008 B & $11.523(.040)$ & $11.022(.042)$ & $10.761(.039)$ & G 188-022 A & $8.984(.021)$ & $8.737(.038)$ & $8.645(.022)$ \\
\hline G 026-009 & $7.770(.018)$ & $7.240(.038)$ & $7.082(.029)$ & G 188-022 B & 13.471(.100) & $13.035(.107)$ & $12.859(.107)$ \\
\hline G 026-010 & $14.894(.086)$ & $15.050(.086)$ & $15.217(.149)$ & G 194-037 A & $13.582(.023)$ & $13.128(.030)$ & $13.044(.029)$ \\
\hline G 027-008 A & $10.213(.023)$ & $9.923(.026)$ & $9.870(.025)$ & G 194 & $15.353(.050)$ & $14.840(.064)$ & $14.472(.075)$ \\
\hline G 027-008 B & $13.942(.056)$ & $13.516(.043)$ & $13.261(.051)$ & G 197 & $6.875(.027)$ & $6.245(.017)$ & $6.059(.017)$ \\
\hline G 028-040 & $9.886(.019)$ & $9.468(.030)$ & $9.407(.021)$ & G 197 & $9.171(.022)$ & $8.659(.021)$ & $8.387(.015)$ \\
\hline LP 581-80 & $11.727(.019)$ & $11.095(.031)$ & $10.922(.018)$ & G 204-049 $\mathrm{A}^{f}$ & $9.426(.023)$ & $9.034(.026)$ & $8.903(.020)$ \\
\hline G 032-046 A & $8.335(.029)$ & $7.928(.051)$ & $7.877(.031)$ & G 204-049 B ${ }^{a}$ & - & - & - \\
\hline G 032- & - & - & - & G 214- & $10.756(.024)$ & $10.443(.030)$ & 10.393 \\
\hline G 059-032 A & 7.401(.034) & $6.953(.067)$ & $6.847(.024)$ & G 214-001 B & 14.511(.100) & $14.077(.089)$ & $13.945(.080)$ \\
\hline G 059-032 B & $14.106(.027)$ & $13.723(.042)$ & $13.215(.033)$ & $45 \mathrm{~A}$ & $9.855(.023)$ & $9.579(.021)$ & $9.496(.019)$ \\
\hline G 061-024 & $7.681(.018)$ & $7.367(.015)$ & $7.302(.017)$ & G 216 & $15.019(.045)$ & $14.454(.049)$ & $14.138(.051)$ \\
\hline G 061- & $8.481(.024)$ & $8.223(.027)$ & $8.120(.023)$ & G 217-004 A & $9.946(.020)$ & $9.569(.018)$ & $9.474(.016)$ \\
\hline G 063-036 $6^{b}$ & $7.643(.023)$ & $7.067(.026)$ & $6.828(.021)$ & G 217 & $14.191(.032)$ & $13.619(.037)$ & $13.397(.038)$ \\
\hline G 066-22 A & $9.030(.0$ & $8.577($. & 8.634 & $7 \mathrm{~A}$ & $8.735(.035)$ & $8.360(.026)$ & $8.297(.021)$ \\
\hline $\mathrm{BD}+062932 \mathrm{~B}$ & $9.304(.095)$ & $8.858(.0$ & $8.816(.0$ & G 230 & $11.745(.024)$ & $11.196(.019)$ & $10.959(.024)$ \\
\hline G 069-004 & $7.035($. & 6.659 & 6.602 & $49 \mathrm{AB}^{c}$ & $7.263(.021)$ & $6.916(.018)$ & $6.866(.020)$ \\
\hline G 069-004 N & $7.790(.020)$ & $7.466(.0$ & $7.397(.031)$ & G 24 & 8.693(.029) & $8.404(.044)$ & $8.360(.021)$ \\
\hline $\mathrm{G} 090-036 \mathrm{AB}^{c}$ & $11.382(.021)$ & $11.046(.022)$ & $10.962(.020)$ & $\mathrm{G} 242$ & $12.214(.025)$ & $11.704(.031)$ & $11.478(.023)$ \\
\hline G 093-027 A & $10.120(.041)$ & $9.610(.046)$ & $9.505(.035)$ & G 251-054 A & $8.711(.039)$ & $8.333(.038)$ & $8.261(.026)$ \\
\hline G 093-027 B ${ }^{d}$ & $11.467(.038)$ & $10.834(.032)$ & $9.463(.055)$ & G 251-054 B & $12.541(.029)$ & $12.027(.035)$ & $11.888(.023)$ \\
\hline G095-057 A & $6.618(.026)$ & $6.192(.021)$ & $6.120(.023)$ & $\mathrm{G} 252-049 \mathrm{AB}^{c}$ & $6.888(.021)$ & $6.242(.020)$ & $6.084(.020)$ \\
\hline G 095-057 B & $6.949(.023)$ & $6.481(.024)$ & $6.347(.023)$ & G 255-038 A & 7.799(.037) & $7.251(.044)$ & $7.108(.024)$ \\
\hline G 116-009 A & $12.660(.021)$ & $12.161(.021)$ & $12.084(.021)$ & G 255-038 B & $9.574(.022)$ & $8.942(.030)$ & $8.709(.022)$ \\
\hline G 116-009 B & $15.331(.045)$ & $14.710(.054)$ & $14.571(.090)$ & G 262-021 & $12.264(.042)$ & $11.769(.044)$ & $11.743(.037)$ \\
\hline G 128-064 A & $8.251(.026)$ & $7.881(.015)$ & $7.836(.019)$ & G 262-022 & $12.240(.029)$ & $11.638(.033)$ & $11.495(.024)$ \\
\hline G 128-064 B & $10.222(.028)$ & $9.763(.026)$ & $9.668(.023)$ & G 266-029 $\mathrm{AB}^{c}$ & $8.263(.018)$ & $8.095(.020)$ & $8.078(.027)$ \\
\hline G 128-077 A & $11.655(.021)$ & $11.154(.024)$ & $11.068(.020)$ & G 273-001 & 7.082(.019) & $6.860(.020)$ & $6.771(.023)$ \\
\hline G 128-077 B & $13.531(.024)$ & $12.960(.026)$ & $12.828(.031)$ & LHS 541 & $13.032(.027)$ & $12.559(.028)$ & $12.414(.026)$ \\
\hline G 153-067 & $8.055(.024)$ & $7.637(.040)$ & $7.517(.024)$ & G $273-152 \mathrm{~A}^{g}$ & $8.694(.026)$ & $8.291(.029)$ & $8.227(.042)$ \\
\hline G 017-027 & $11.086(.022)$ & $10.541(.022)$ & $10.319(.019)$ & G 273-152 $\mathrm{B}^{a}$ & - & - & - \\
\hline G 171-050 A & $11.939(.023)$ & $11.640(.022)$ & $11.603(.023)$ & $\mathrm{BD}+162116 \mathrm{AB}^{c}$ & $5.894(.021)$ & $5.550(.016)$ & $5.467(.021)$ \\
\hline G 171-050 B & $15.909(.060)$ & $15.452(.087)$ & $15.191(.126)$ & $\mathrm{BD}+232207 \mathrm{~A}$ & $4.995(.260)$ & $4.696(.228)$ & $4.512(.017)$ \\
\hline G 172-016 A & $9.748(.023)$ & $9.420(.020)$ & $9.333(.018)$ & $\mathrm{BD}+232207 \mathrm{~B}$ & $8.355(.026)$ & $7.794(.047)$ & 7.593(.026) \\
\hline
\end{tabular}

${ }^{a}$ No 2MASS photometry available.

${ }^{b}$ Combined photometry of G 063-036 and GJ 516 B.

${ }^{c}$ Combined photometry.

${ }^{d}$ Error in $K_{\mathrm{s}}$ : the detection was not geometrically possible.

${ }^{e} J H$ are upper limits to the photometry. $K_{\mathrm{s}}$ is derived from a profile-fitting technique.

${ }^{f}$ Magnitudes are derived from a profile fitting measurement. Not likely contaminated by the companion.

${ }^{g}$ Photometry is partially contaminated by the companion.

similar types) but detectable $\mathrm{TiO}$ absorption at around $710 \mathrm{~nm}$. Also noticeable are the atomic features ascribed to Na I, K I and $\mathrm{Ca}$ II. We checked for the presence of the $\mathrm{H} \alpha$ line. At the resolution of our spectra, $\mathrm{H} \alpha$ is not seen in emission in any source. Hence, there is no evidence of chromospheric activity, as expected for old, metal-poor stars. Additionally, this suggests that none of them are interacting close binaries.

\subsubsection{Spectral typing}

We followed the classification scheme developed by Reid et al. (1995), Gizis (1997) and Lépine et al. (2003a) to obtain the spectral type of our objects. This scheme is based on the molecular indices $\mathrm{TiO} 5, \mathrm{CaH} 2$ and $\mathrm{CaH} 3$, which are excellent indicators of temperature and metallicity. The $\mathrm{CaH} 2$ and 
Table 5. Log of spectroscopic observations.

\begin{tabular}{lcccc}
\hline \hline Object & Obs. date & $\begin{array}{c}\text { Exp. } \\
(\mathrm{s})\end{array}$ & $\begin{array}{c}\Delta \lambda \\
(\AA)\end{array}$ & $\begin{array}{c}\text { Coverage } \\
(\mathrm{nm})\end{array}$ \\
\hline \multirow{5}{c}{ WHT spectra } \\
BD+06 2932 B & 2 Jul. 1994 & 300 & 6.1 & $386-590$ \\
& 2 Jul. 1994 & 300 & 5.7 & $605-900$ \\
G 116-009 B & 1 Jul. 1994 & 300,600 & 6.1 & $386-590$ \\
& 1 Jul. 1994 & 300 & 5.7 & $605-900$ \\
G 128-077 B & 23 Aug. 2002 & $2 \times 400$ & 3.3 & $391-580$ \\
& 23 Aug. 2002 & $2 \times 400$ & 3.4 & $641-775$ \\
G 172-016 B & 11 Sep. 2003 & $2 \times 600$ & 6.2 & $575-1000$ \\
G 176-046 D & 2 Jul. 1994 & $2 \times 600$ & 6.1 & $386-590$ \\
2 Jul. 1994 & $2 \times 600$ & 5.7 & $605-900$ \\
G 188-022 B & 11 Sep. 2003 & $3 \times 600$ & 6.2 & $575-1000$ \\
G 214-001 B & 11 Sep. 2003 & $3 \times 600$ & 6.2 & $575-1000$ \\
LHS 541 & 23 Aug. 2002 & $3 \times 300$ & 3.3 & $391-580$ \\
& 23 Aug. 2002 & $3 \times 300$ & 3.4 & $641-775$ \\
& 11 Sep. 2003 & 600 & 6.2 & $575-1000$ \\
\hline \multicolumn{5}{c}{ TNG spectra } \\
G 216-045 B & 12 Dec. 2003 & $2 \times 900$ & 13 & $525-900$ \\
G 273-152 B & 12 Dec. 2003 & 60 & 13 & $525-900$ \\
\hline
\end{tabular}

$\mathrm{CaH} 3$ indices measure the strength of the $\mathrm{CaH}$ bands blueward of $705 \mathrm{~nm}$, and the TiO5 index measures the $\mathrm{TiO}$ band head redward of $705 \mathrm{~nm}$. Gizis (1997) claimed that the $\mathrm{CaH} 3$ index is particularly well suited for the "numerical" classification of metal-poor and extreme metal-poor dwarfs in the range K7-M6. Classes are K7, M0, M1 and so on. The measured indices for our stars are given in Table 6; the uncertainty is about $5-10 \%$. We also provide the final averaged spectral types obtained to an accuracy of \pm 0.5 subclasses. We note, however, that the spectral classes of G 216-045 B and G 273-152 B may suffer from larger error bars, i.e., one subclass, because they were not observed at parallactic angle. We adopted the nomenclature widely used in the literature, and spectral types have the prefix "sd" or "esd" that stand for "subdwarf" or "extreme subdwarf", respectively. All of our WHT spectroscopically observed objects are extreme subdwarfs according to their very low metallicities. The TNG stars are classified as "sdM" because of their relatively high metallicity. Our spectral measurements of the objects in common with Gizis \& Reid (1997), i.e., LHS 541, G 116-009 B, and G 176-046D, are fully consistent with previously published values. The new companions have spectral classes ranging from very late- $\mathrm{K}$ to early- $\mathrm{M}$, which is in agreement with their optical and near-infrared colors.

The spectral types of G 172-016B and G214-001 B are quite alike within the error bars. However, a detailed inspection of the red spectra of Fig. 3 reveals that the intensity of the molecular bands at around 620 and $680 \mathrm{~nm}$ is different: G 214-001 B shows less molecular absorption. This is explained by the more metal-depleted atmosphere of this object. Because they have similar metallicity $([\mathrm{Fe} / \mathrm{H}] \sim-1.5)$, G 172-016 B, G 116-009 B, G 188-022 B and LHS 541 are better examples of the noticeable effect of decreasing effective temperature in metal-deficient dwarfs: the $\mathrm{NaI}$ and K I resonance doublets, and all molecular absorptions become stronger at lower temperatures. However, and despite the fact that the
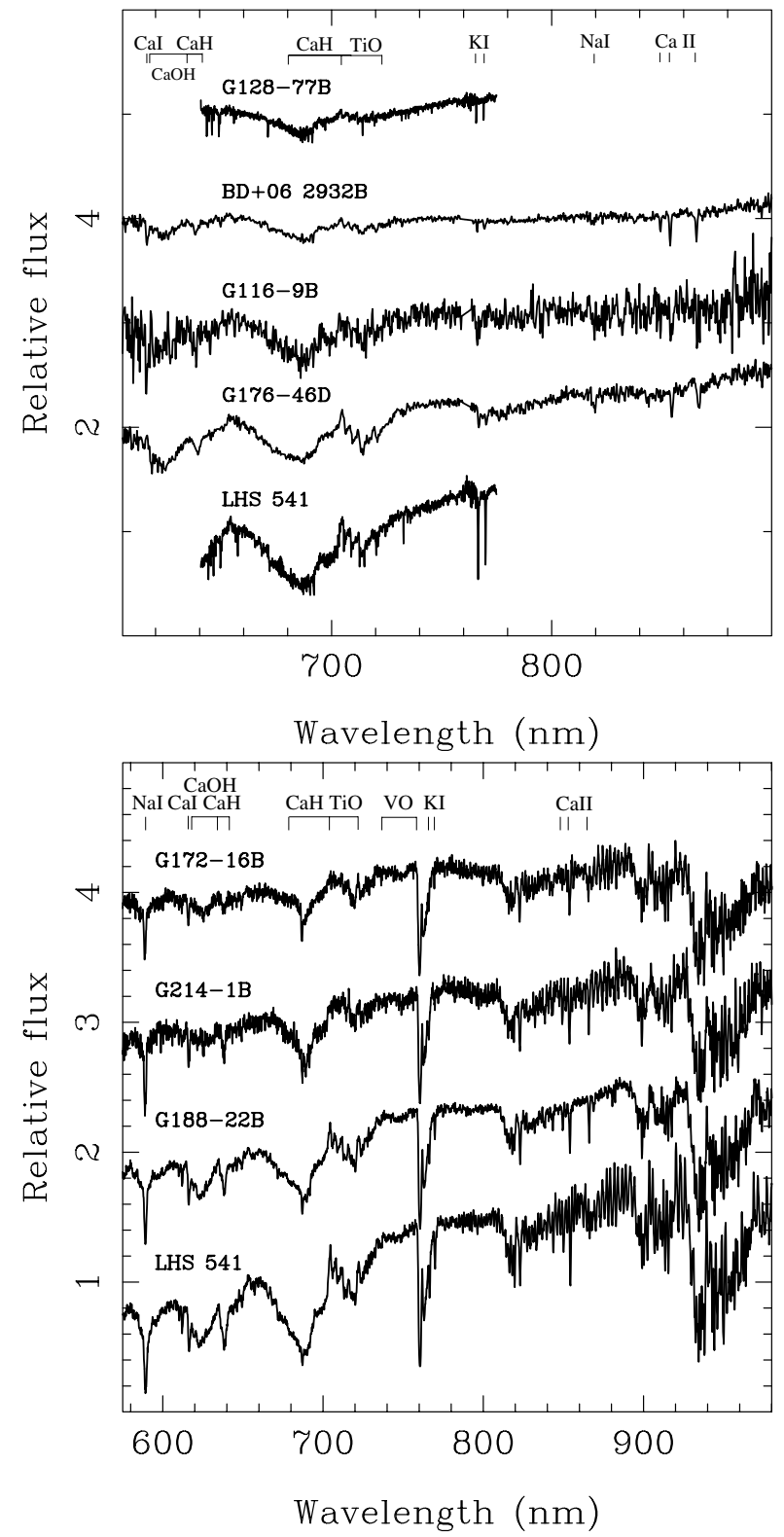

Fig. 3. WHT spectra of subdwarfs ordered by decreasing spectral type from top to bottom. Data are normalized to unity at $660-662.5 \mathrm{~nm}$, and are displaced upwards by 1 for clarity. The top panel displays spectra corrected for telluric absorptions. The bottom panel shows spectra with strong telluric features around $687,718,760,815,900$, and $932 \mathrm{~nm}$. Fringing is observed redwards of $800 \mathrm{~nm}$. Some spectral features are identified at the top of each panel.

objects shown in Fig. 5 have quite different classes, their blue spectroscopic data appear rather similar, except for the slightly larger slope towards red wavelengths of LHS 541, which is the latest type subdwarf. We note that red spectra $(550-800 \mathrm{~nm})$ are preferred over blue spectra $(400-550 \mathrm{~nm})$ for the spectral classification of low-metallicity stars, since the red spectroscopic features are more sensitive to temperature changes than the blue features. 


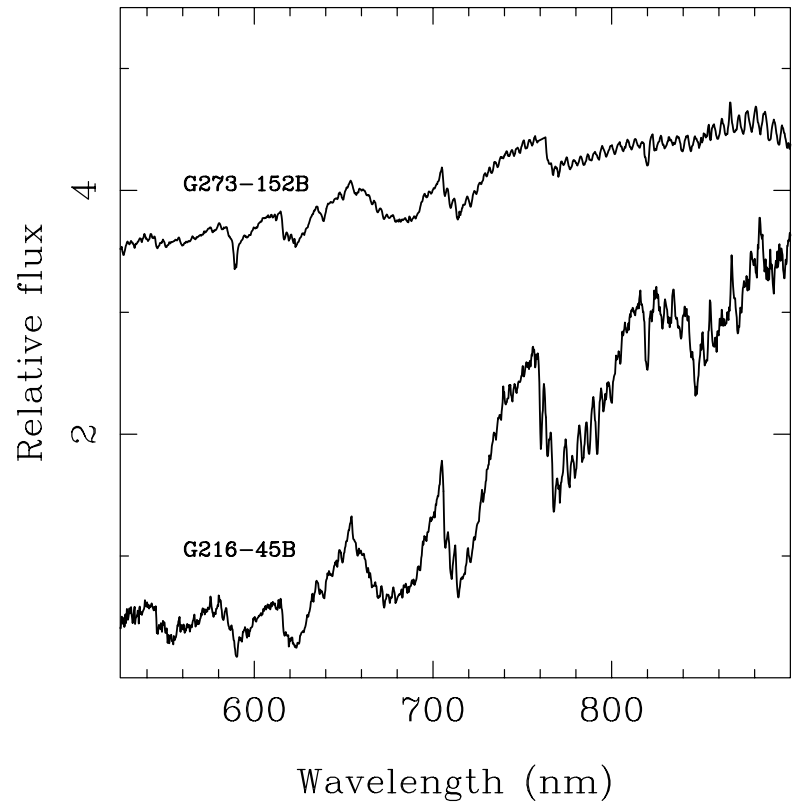

Fig. 4. TNG spectra of slightly metal-poor, M-type proper motion companions. Data are normalized to unity at $660-662.5 \mathrm{~nm}$, and are displaced upwards by 3 for clarity. Note that these spectra were not taken at parallactic angle; hence, the flux ratio between very red and blue wavelengths may be incorrect. Telluric absorption and fringing are removed.

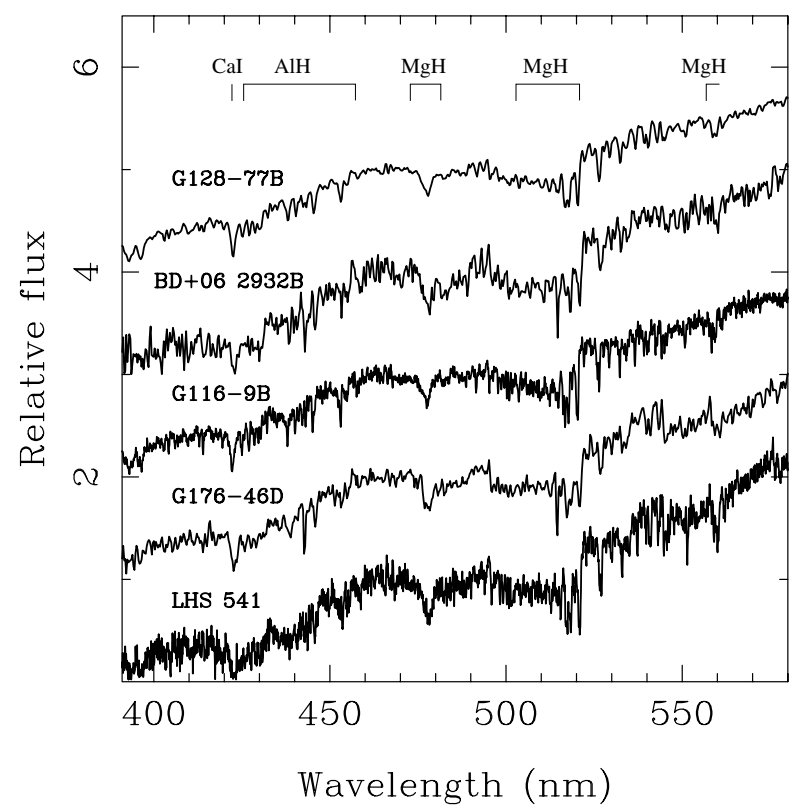

Fig. 5. WHT blue spectra normalized to unity at $461-463 \mathrm{~nm}$, and offset upwards by 1 for clarity. Spectral features are identified following Ake \& Greenstein (1980) and Dahn et al. (1995).

\section{Discussion}

From our survey, we found 13 new common-proper-motion pairs, and retrieved a total of 29 known pairs. This suggests that about one third of the stellar wide companions were missed in previous proper motion searches.
Table 6. Spectroscopic data.

\begin{tabular}{lcccc}
\hline \hline Object & TiO5 & CaH2 & CaH3 & $\begin{array}{c}\text { SpT } \\
( \pm 0.5)\end{array}$ \\
\hline BD+06 2932 B & 0.872 & 0.819 & 0.900 & esdK7.3 \\
G 116-009 B & $0.9:$ & 0.726 & 0.869 & esdM0.0 \\
G 128-077 B & 0.928 & 0.823 & 0.913 & esdK7.2 \\
G 172-016 B & 0.902 & 0.818 & 0.860 & esdK7.5 \\
G 176-046 D & 0.709 & 0.622 & 0.809 & esdM1.0 \\
G 188-022 B & 0.813 & 0.621 & 0.764 & esdM1.0 \\
G 214-001 B & $1.0:$ & 0.765 & 0.829 & esdM0.0 \\
G 216-045 B & 0.526 & $0.42:$ & 0.713 & sdM3.0 \\
G 273-152 B & 0.717 & 0.656 & 0.838 & sdM1.0 \\
LHS 541 & 0.731 & 0.469 & 0.641 & esdM3.0 \\
\hline
\end{tabular}

Notes: a semicolon indicates error bars twice larger than those given in the text. The uncertainty in the spectral classification of G 216-045 B and G 273-152 B may be one subclass.

\subsection{Photometric diagrams}

The optical and near-infrared photometry of Tables 2-4 suggests that most of the proper motion companions are dwarfs. There are two exceptions: G026-010, which is a known white dwarf companion to the K3-type star G026009 (Wegner 1973), and G032-046B, which is companion to a G8-type star (Allen et al. 2000). G 026-010 shows quite blue optical and 2MASS colors that are typical of normal, hot white dwarfs. However, G 032-046 B appears redder, with $M(V)=14.30 \pm 0.15 \mathrm{mag}$ and $(V-I)=0.84 \pm 0.15 \mathrm{mag}$. Despite its relatively brightness in the visible, it is not detected by 2MASS $(J \geq 17)$, which indicates that $\mathrm{G} 032-046 \mathrm{~B}$ is indeed a "blue" object $(I-J \leq 0.8)$. In fact, it is listed in the cata$\log$ of white dwarfs by Luyten (1970). In a $M(V)$ vs. $(V-I)$ diagram like that of Fig. 10 of Monet et al. (1992), G 032-046 B lies at the bottom of the degenerate sequence. Hence, this object is a cool, low-metallicity white dwarf.

Figure 6 depicts the optical color-magnitude diagram of the proper motion companions with $V I$ photometry. The two white dwarfs are labelled. To transform observed magnitudes into absolute magnitudes we used the distance to the primary stars from the Hipparcos database. If no Hipparcos data were available, we used the values given by Carney et al. (1994) and Laird et al. (1988). We assumed that the distance to the companions is equal to the distance to their primaries. In the figure we plotted different symbols for different metallicities. Because no calibrated photometry in the $V, R$ and $I$ bands is available for all of the objects, the $x$-axis of Fig. 6 represents the $(V-I)$ color difference $[\Delta(V-I)]$ between the companion and its primary star. We note that this quantity is reddening-free. As expected, white dwarfs delineate a degenerate sequence at low luminosities, and dwarfs extend to very red colors. Dwarf companions that are fainter than their primary stars are also redder.

The observed scatter in the dwarf sequence of Fig. 6 is very likely due to the different colors of the primaries (which have an effect on the $\Delta(V-I)$ values), unresolved binarity, photometric errors, uncertainties in the distance determination, and different degrees of atmospheric metallic composition. Despite all these uncertainties, it is obvious that the most metal-rich stars 

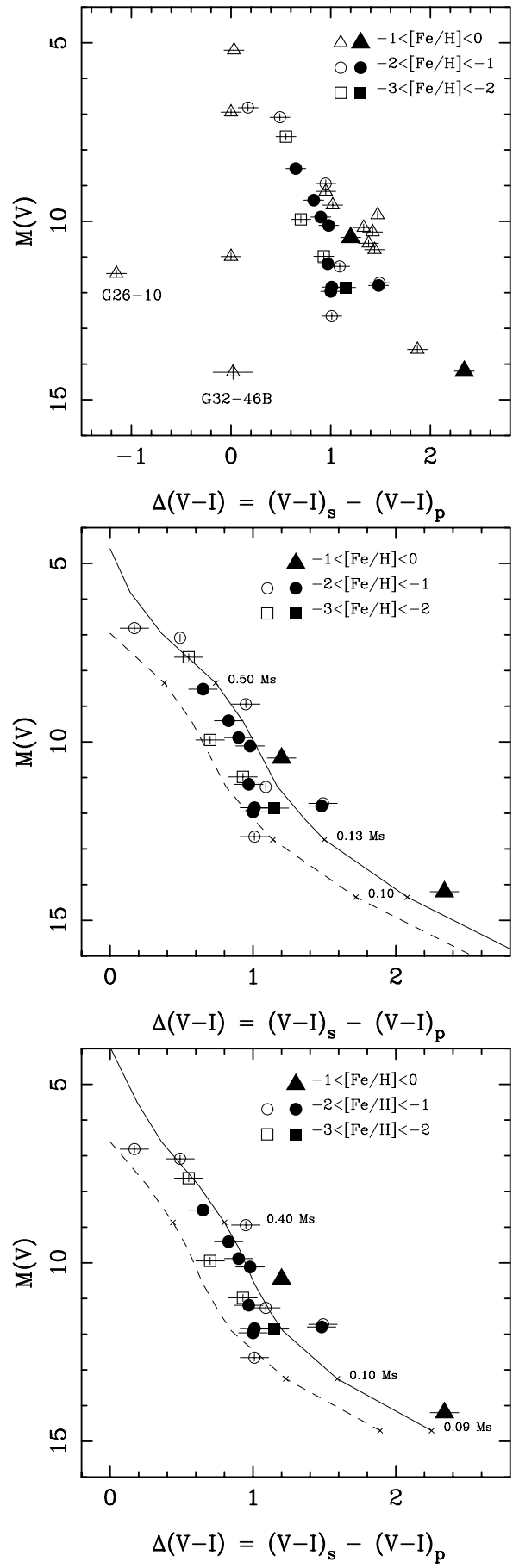

Fig. 6. Top panel: optical color-magnitude diagram of proper motion companions. Different symbols denote different intervals of metallicity (in logarithmic scale), as indicated within the figure. Filled symbols stand for the new discoveries, and open symbols represent previously known secondaries. Middle panel: model calculations by Baraffe et al. (1997) for metallicity -1 dex are overplotted. The track with $(V-I)$ color difference computed for a primary mass of $0.8 M_{\odot}$ is depicted with a full line, and that computed for a primary mass of $0.6 M_{\odot}$ is displayed with a dashed line. Bottom panel: as in the previous panel, but for a metallicity of $-2 \mathrm{dex}$.

appear brighter for a given color. This is due to the fact that most of the primary stars have similar optical colors (spectral types F8-K0), and that metal-depleted stars are more subluminous for lower metallicities. Besides the two white dwarfs, there are two outliers in Fig. 6: GJ 516 B and G 19750 , which appear very faint for their colors. Their primary stars are significantly redder (spectral type $\mathrm{M}$ ) than other primaries.

To compare the location of the observed photometric sequence of metal-poor dwarf companions to theoretical models, we overplotted 10-Gyr isochrones from Baraffe et al. (1997) in the middle and bottom panels of Fig. 6. Here, we did not display known secondaries with $[\mathrm{Fe} / \mathrm{H}]>-1$ for clarity. This has no impact on the following discussion. The metallicities of the theoretical tracks depicted in the figure are -1 and -2 dex; the majority of the new proper motion companions have a metal content within this range. According to Carney et al. (1994), the mass of the primary stars ranges from 0.55 to $0.8 M_{\odot}$, the mean value being $0.67 M_{\odot}$. We note, however, that the most metalrich primaries have larger masses than the stars with lower metal abundance. To compute the predicted $\Delta(V-I)$ color differences, we considered masses of 0.6 and $0.8 M_{\odot}$, i.e., two stars of the same mass and metallicity have $\Delta(V-I)=0.0$ mag, and the color difference increases for smaller mass ratios. As seen from Fig. 6, the models nicely reproduce the trend described by the observations. Baraffe et al.'s models also reproduce reasonably well the entire main sequence of globular clusters.

We also produced the 2MASS near-infrared colormagnitude diagram shown in Fig. 7. As in the previous figure, the 10-Myr isochrones from Baraffe et al. (1997) are overplotted. These authors provide near-infrared colors in the CIT photometric system. To convert them into the 2MASS reference system, we used the transformation equations given in Carpenter (2001). As in the visible, the agreement between the trend delineated by near-infrared observations and theory is noticeable.

The near-infrared two-color diagram is depicted in Fig. 8. In the upper panel, the sequence of field, solar-metallicity stars from Bessell \& Brett (1988) is overplotted. We adopted the color transformations of Alonso et al. (1994) to convert Bessell \& Brett's data to the TCS system, which is very similar to the 2MASS photometric system (see Béjar et al. 2003). The lower panel of Fig. 8 displays an enlargement of the color-color diagram, where the two isochrones of Baraffe et al. are considered and error bars are avoided for the sake of clarity. From this picture, metallicity effects become apparent: the $(J-H)$ colors of the most metal-deficient companions (squares) appear blueshifted as compared to the colors of the most metalrich stars (triangles), indicating that metallicity has a clear effect on the near-infrared colors. This signature is qualitatively reproduced by the models. Further discussion on the colors of subdwarfs and dwarfs is provided in Leggett (1992) and Tinney et al. (1993). Also remarkable is the theoretically predicted turn-over to the blue (both $J-H$ and $H-K$ ), which is due to the increasing collision-induced absorption of molecular hydrogen with increasing density and decreasing temperature (Saumon et al. 1994). This turn-over takes place at around $0.2 M_{\odot}$ and $T_{\text {eff }}=3600-4000 \mathrm{~K}$ for the two metal-depleted abundances. A similar feature is observed in solar-metallicity dwarfs (e.g., Leggett et al. 2002, and references therein), but it occurs at much lower temperatures $\left(T_{\text {eff }} \sim 1500 \mathrm{~K}\right)$, within the 


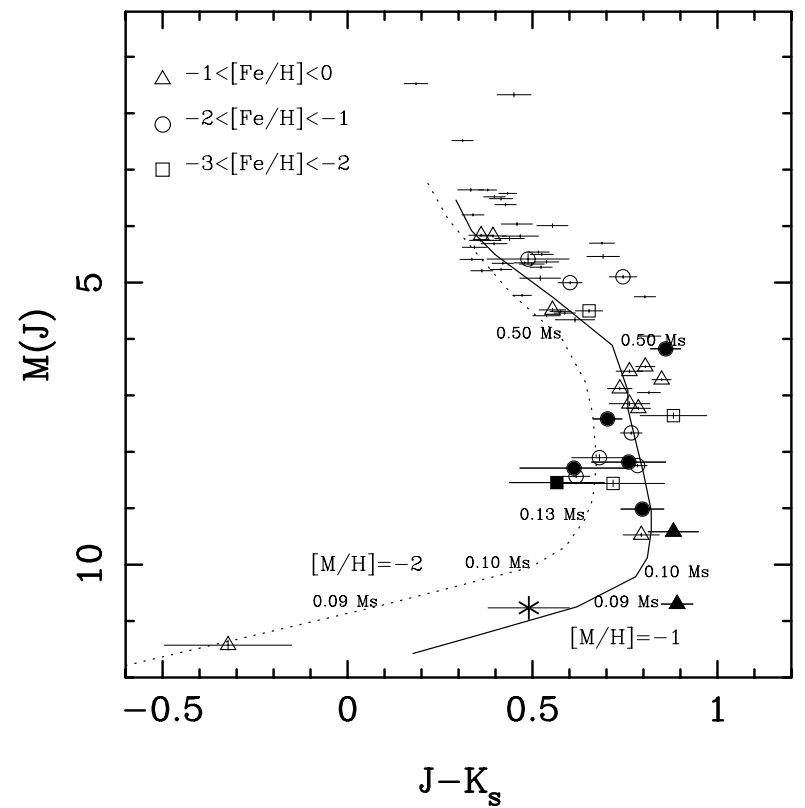

Fig. 7. 2MASS near-infrared color-magnitude diagram for the wide binaries in our sample and the esdM8.0 star LSR 1425+7102 (asterisk). Primary stars are plotted as error bars without any symbol. New companions are plotted with filled symbols. The object with the bluest color is a white dwarf. Overplotted onto the data are the 10-Myr isochrones from Baraffe et al. (1997). The faint end of the isochrones indicates the location of the substellar limit for each metallicity.

substellar mass domain, and is explained by the appearance of strong methane absorption at 1.6 and $2.2 \mu \mathrm{m}$.

Three proper motion companions show much redder near-infrared colors than expected for their metallicity and brightness: G 262-022 $([\mathrm{Fe} / \mathrm{H}]=-1.07), \mathrm{G} 009-047 \mathrm{~B}$ $([\mathrm{Fe} / \mathrm{H}]=-1.93)$, and $\mathrm{G} 194-037 \mathrm{~B}([\mathrm{Fe} / \mathrm{H}]=-2.03)$. Except for $\mathrm{G} 059-032 \mathrm{~B}, \mathrm{G} 009-047 \mathrm{~B}$ and $\mathrm{G}$ 194-037 B have the reddest $(J-H)$ and $\left(H-K_{\mathrm{s}}\right)$ colors, respectively, in our final list of companions (see Figs. 6 and 7). These objects appear shifted to the red by more than $2-\sigma$ their photometric uncertainties provided their atmospheric chemical composition is accurately determined. The color excesses observed in G 262-022 and G009-047 B could be explained by small amounts of interstellar reddening $\left(A_{V} \sim 0.3-1 \mathrm{mag}\right)$, as illustrated by the reddening vector in the two panels of Fig. 7. A fainter companion might also be contributing to the very red $\left(J-K_{\mathrm{s}}\right)$ color of G 262-022. Carney et al. (1994) detected some extinction in G 262-021 (the primary star), but no extinction has been photometrically measured in G 009-047 A (Laird et al. 1988). The $K_{\mathrm{s}}$-band excess of $\mathrm{G} 194-037 \mathrm{~B}$ is not compatible with interstellar reddening alone (no extinction is measured by Carney et al. 1994). From the models, less massive, unresolved companions are not expected to contribute significantly to the nearinfrared flux excess below $M(J)=6 \mathrm{mag}$, since the theoretical photometric sequence goes down to $M(J)=10$ mag almost vertically in the $J$ vs. $\left(J-K_{\mathrm{s}}\right)$ diagram of Fig. 6 . There are two possibilities: either there is a warm dusty disk surrounding G 194$037 \mathrm{~B}$, or its low metallicity is overestimated (most likely). We note that the very recent work by Latham et al. (2002) gives $[\mathrm{Fe} / \mathrm{H}]=-1.0$ for $\mathrm{G} 194-037 \mathrm{~A}$ (the primary star). This is in
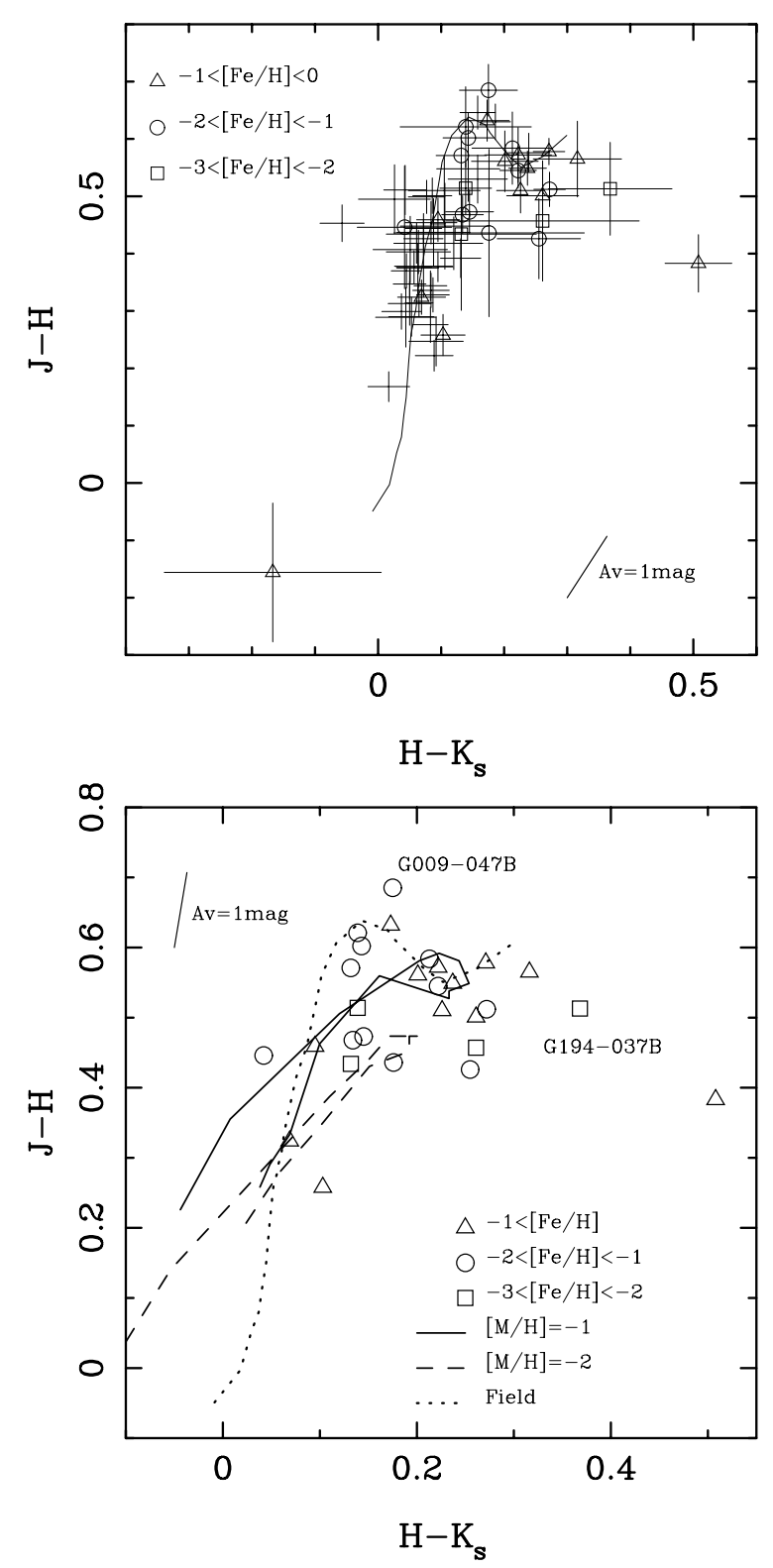

Fig. 8. Top panel: 2MASS color-color diagram for the wide binaries in our sample. Both new and known companions are plotted with open symbols. Primary stars are plotted as error bars without any symbol. Overplotted onto the data is the field dwarf sequence from Bessell \& Brett (1988). The object with the bluest color is a white dwarf. Bottom panel: detail of the color-color diagram where models (solid and dashed lines) calculated for two different metallicities are overplotted (Baraffe et al. 1997). Error bars are omitted for clarity. The reddening vector corresponding to $A(V)=1$ mag (Rieke \& Lebofski 1985) is plotted in every panel.

better agreement with the location of the proper motion companion in Fig. 7.

\subsection{Mass estimate}

An estimate of the mass of the proper motion secondaries can be obtained from the comparison of the loci of the objects in color-magnitude diagrams with evolutionary tracks. As inferred from Figs. 6 and 7, the new companions have stellar 
masses between 0.1 and $0.5 M_{\odot}$, as have many of the previously known companions of our survey and field metal-depleted stars (Monet et al. 1992).

One of the reddest, least massive, low-metallicity stars detected in our work is G 216-045 B. The companion G059$032 \mathrm{~B}$ is actually the reddest (mid-M spectral class according to its near-infrared colors), but its metallicity (assumed to be equal to that of the primary star) is very close to solar. According to Figs. 6 and 7, the mass of G 216-045 B can be estimated at $0.1-0.13 M_{\odot}$ if we adopt an age typical of the old disk. The spectral type of G216-045 B is sdM3 on the basis of its optical spectrum. This star actually belongs to a multiple system formed by at least three members. The primary star, G 216045 A (spectral type K1, Lee 1984), is found to be a 1153 dayperiod spectroscopic binary by Latham et al. (2002). The projected semi-major axis of the spectroscopic double is $112.8 \mathrm{AU}$, while G 216-045 B is separated by about 4150 AU (see Table 3) from the more massive stars. Multiple systems with wide companions are not rare among metal-deficient stars, as discussed in Martín et al. (1995).

Some later M-type subdwarfs have been recently identified in the field by means of proper motion measurements. Lépine et al. (2003b) reported on the finding of a low-metallicity star with spectral type sdM8.0: LSR 1425+7102. For comparison purposes, we plot its 2MASS photometry as an asterisk in Fig. 7. Its location in the figure suggests that the atmospheric metal abundance of this star is around $[\mathrm{M} / \mathrm{H}]=-1$ (i.e., all metals are depleted by a factor of 10 as compared to the Sun). From the comparison with model calculations, its mass is likely between 0.085 and $0.09 M_{\odot}$, rather close to the substellar borderline for this metallicity (i.e., $0.083 M_{\odot}$, Baraffe et al. 1997). We do not detect in our survey objects as cool as this one because the images are not deep enough (short exposure times).

Nevertheless, on the basis of our results, we remark that the observational strategy described in this paper can lead to the detection of metal-poor brown dwarf companions. The substellar borderline occurs at $M(I)=13.93 \mathrm{mag}$ and $M(J)=11.57 \mathrm{mag}$ for $[\mathrm{M} / \mathrm{H}]=-1$ and age $=10 \mathrm{Gyr}$ (Baraffe et al. 1997; Chabrier $\&$ Baraffe 1997). For younger ages, the star-brown dwarf frontier occurs at brighter magnitudes. The most massive brown dwarfs are expected to be about 1 mag fainter in $J$ and to show cool atmospheres with temperatures below $1000 \mathrm{~K}$; their peak of emission will lie mostly between the $R$ and $H$ wavelengths (Saumon et al. 1994; Allard \& Hauschildt 1995). These values are within the capabilities of mid-size telescopes and their optical and near-infrared instrumentation. As compared to solarmetallicity brown dwarfs of similar mass, the models predict that low-metallicity substellar objects are significantly bluer in the infrared colors (see the turn-over of the isochrones in Figs. 7 and 8).

\subsection{Wide binaries, metallicity and frequency}

Figure 9 illustrates the projected separation of the commonproper-motion companions to G-, K- and M-type subdwarfs as a function of metallicity. Numerical values are provided in Tables 2 and 3. Separations range from a few tens of $\mathrm{AU}$

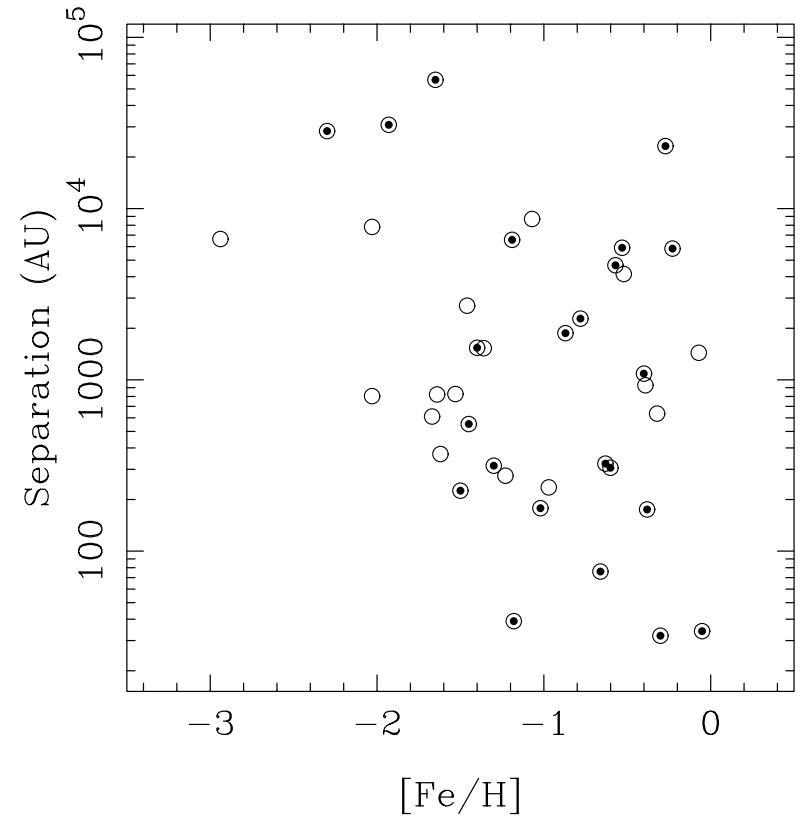

Fig. 9. Projected separation between companion and primary star against metallicity. Encircled dots stand for stars with Hipparcos distances.

up to about $57000 \mathrm{AU}$. The lack of small orbits at very low metallicities is very likely an observational effect due to the distance-to-the-star distribution shown in Fig. 2, and should not be considered reliable. Metal-poor, low-mass stars are found in wide binary systems with orbital sizes that resemble those of solar-metallicity binary stars of similar masses (e.g., Gliese \& Jahreiss 1988; Poveda et al. 1994). This result agrees with the very recent and extensive work of Latham et al. (2002), who reported finding 188 spectroscopic binaries in a sample of 1359 stars selected from the Carney-Latham proper-motion surveys. These authors concluded that there is no obvious discrepancy between the close binary characteristics in the halo and the disk populations. Concerning wide systems, we do not find significant difference in the orbital separations of metaldepleted binaries and solar-metallicity multiple stars.

About $9 \%$ of the metal-poor G-, K- and M-type stars in our sample have visual, common proper motion stellar companions in wide orbits $(\geq 30 \mathrm{AU})$ and with mass ratios of $0.13-1.0$. A similar binary fraction was obtained by Allen et al. (2000) after examining more than 1200 low-metallicity stars from the catalog of Schuster \& Nissen (1989). Other surveys of metal-deficient, high-velocity stars yielded slightly higher frequencies $(\sim 13 \%)$, but they are all based on small statistics (e.g., Abt \& Willmarth 1987; Martín \& Rebolo 1992). Poveda et al. (1994) provided a catalog of wide binaries and multiple stars in the solar vicinity with separations larger than $25 \mathrm{AU}$. The great majority of the stars in their catalog (98.5\%) has solar metallicity. According to these authors, the fraction of wide binaries in the solar neighborhood is $15-20 \%$, which is about a factor of 2 larger than the one found for low-metallicity stars.

However, we will show that this "apparent" low frequency of metal-deficient wide companions is likely due to the undersampling of the smallest orbits at very low metallicities. 


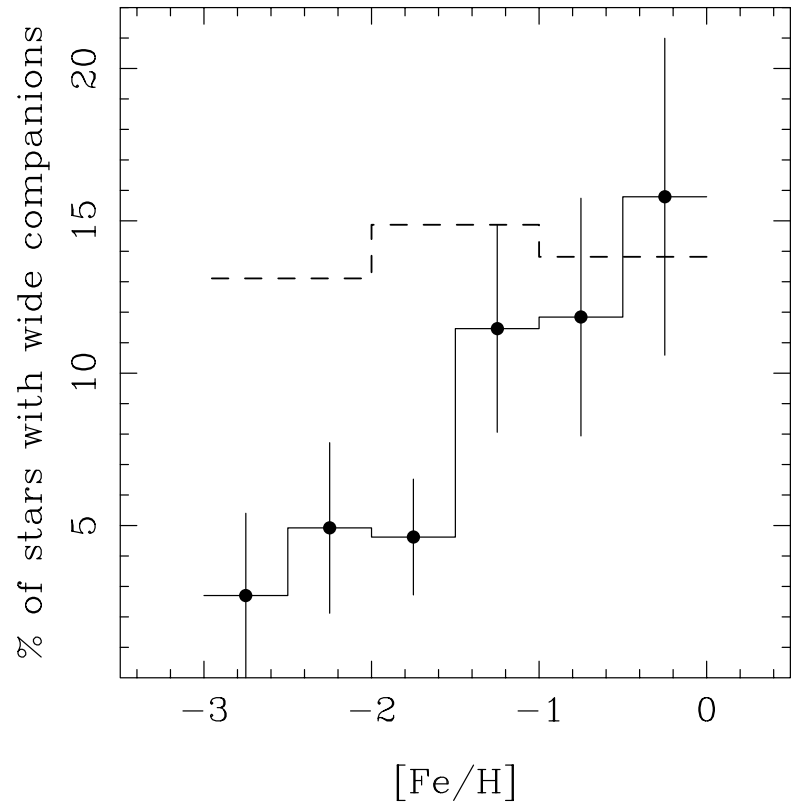

Fig. 10. The frequency of low-metallicity wide companions is plotted as a solid line. The bin width represents a change of 0.5 dex in the metal logarithmic abundance. Vertical errors are Poissonian. The "corrected" frequency is plotted as a dashed line (see text), where the bin width is 1 dex.

The metallicity distribution of our companion stars (thick line) is compared to the distribution of the sample targets in Fig. 1. We calculated the cumulative metallicity distributions of both datasets and applied the Kolmogorov-Smirnov test to investigate the degree of similarity. We obtained a probability of 0.42 . Such a low value indicates that the two distributions are different. To further illustrate this, we evaluated the binary fraction of stars with visible companions in our survey as a function of metal content. For binary fraction, we used the number of multiple systems relative to the total number of stars per interval of metallicity. The results are depicted in Fig. 10 (solid line). From the picture, there is a marked decrease toward very low metallicities: while $12-16 \%$ of the most metal-rich stars $([\mathrm{Fe} / \mathrm{H}] \geq-1)$ harbor wide companions, less than $5 \%$ of the most metal-deficient stars $([\mathrm{Fe} / \mathrm{H}] \leq-2)$ are found in wide binaries.

Nevertheless, Fig. 10 needs to be corrected for the distancemetallicity relation shown in Fig. 2. The most metal-depleted stars in our survey are located farther away than the most metalrich stars. Hence, we explored the widest orbits of the very lowmetallicity stars, while closer regions were surveyed around more metal-rich sources. We corrected the "direct" binary fraction for this effect by taking into account the different orbital sizes that our survey has explored as a function of metallicity, and normalizing at $[\mathrm{Fe} / \mathrm{H}]=[-1,0]$. The resulting "corrected" binary frequency is plotted as a dashed line in Fig. 10. We note that this correction is based on the assumption that companions have similar orbits throughout all metallicities. This assumption is supported by Fig. 9, where it is obvious that this assessment is true for the interval $[\mathrm{Fe} / \mathrm{H}]=[-1.5,0]$. The corrected binary fraction is nearly flat, and suggests that 13$15 \%$ of the low-metallicity stars harbor wide, low-mass stellar companions with mass ratios in the range $0.13-1.0$ and separations larger than $30 \mathrm{AU}$. This overall binary frequency is in agreement with the binary fraction observed among main sequence G- to M-type stars in the solar neighbourhood (Poveda et al. 1994) and young T Tauri stars of star-forming regions $(14 \pm 1.8 \%$, Brandner et al. 1996).

Of the total of 42 pairs, at least 11 are multiple systems ( 26\%). The star G 273-001 is resolved to be double by Hipparcos $\left(\rho=0 .{ }^{\prime} 816, \mathrm{PA}=226 \mathrm{deg}, \Delta V=0.22 \mathrm{mag}\right)$. The primary stars G059-032 A (Carney et al. 1994), G 069-004 (Carney et al. 1994), G 061-024 (Goldberg et al. 2002), G 128064 A (Latham et al. 2002), G 153-067 (Carney et al. 1994), G 173-002 (Latham et al. 2002), G 116-009 A (Latham et al. 2002), G 176-046 A (Latham et al. 1992), and G216045 A (Latham et al. 2002) are found to be spectroscopic binaries. The companion G 095-057 B is a single-lined system with orbital solution according to Carney et al. (1994). The low-metallicity stars G 009-047 A and G 214-001 A also show quite large dispersions in their radial velocity measurements (Latham et al. 2002), but no conclusion on their spectroscopic binary nature can be reached so far. Martín et al. (1995) noted that the new companion G 176-046D is part of a multiple system formed by 4 components whose masses range from 0.13 up to $0.7 M_{\odot}$. The total fraction of low-metallicity multiple systems composed of three or more members cannot be derived because not all the stars have been investigated for radial velocity variations or imaged to very high spatial resolutions (e.g., adaptive optics). We can simply derive a lower limit from our survey, which is $2.3 \%$. It is a very intriguing question how these multiple systems formed and how they survived dynamical evolution.

We note that the $13-15 \%$ wide binary frequency observed among low-metallicity G- to M-type stars is also comparable to the fraction of close (unresolved) binaries, which is $\sim 14 \%$ according to the spectroscopic work by Latham et al. (2002). Furthermore, many of the low-mass companions are separated from their primary stars by more than $100 \mathrm{AU}$. If metaldepleted double or multiple systems are formed at birth, this suggests that they were not subjected to the very severe dense conditions of globular clusters for very long (Ryan 1992). On the contrary, their existence in significant numbers suggests that they were likely formed in more relaxed environments. The similarity between the incidence of binaries among lowmetallicity and solar-metallicity stars also suggests that the chemical composition of the parental molecular cloud has little influence over the stellar formation of wide binary and multiple systems. This may also apply to substellar formation. Solar-metallicity brown dwarfs are found as companions to stars in a great variety of orbital sizes: from a few tens of AU (e.g., Nakajima et al. 1995), to several hundred AU (e.g., Rebolo et al. 1998), and to several thousand AU (e.g., Burgasser et al. 2000). The search for metal-poor substellar counterparts is the natural extension of the present work.

\section{Summary and conclusions}

We explored the nearby regions around 473 low-metallicity Gto M-type stars searching for low-mass stellar companions that 
orbit their primary stars at wide separations (typically $\geq 30$ AU). The great majority of the target stars, with high proper motions and metallicities in the range $[\mathrm{Fe} / \mathrm{H}]=[-3.5,0.0]$, were selected from Carney et al. (1994) and Laird et al. (1988). All of them were imaged in the $I$-band with a 1 -m class telescope. Optical $V$ and $R$ data were also collected for a large number of the targets. The dynamical range of the CCD detectors allowed us to detect companions up to 5 mag fainter (completeness) than the target star. The physical link between a candidate and its primary star was assessed by means of photometric and proper motion measurements. We also searched the literature and various archives to complete our survey. We identified 13 new proper motion companions and retrieved 29 previously known companions. This suggests that about one third of the low-mass companions were missed in previous proper motion searches.

2MASS $J H K_{\mathrm{S}}$ photometry is provided for a total of 39 companions out of 42 . On the basis of optical and nearinfrared colors, the 13 new companions are dwarfs. Two out of the 29 previously known companions are white dwarfs. We produced optical and near-infrared color-magnitude and colorcolor diagrams, and overplotted low-metallicity models from Baraffe et al. (1997). The agreement between observations and theory is reasonable, indicating that dwarf companions have masses in the range $0.1-0.5 M_{\odot}$.

Low-resolution optical spectra from 386 to $1000 \mathrm{~nm}$ were obtained for 8 of the new proper motion companions, for which we derived subdwarf spectral types esdK7.2-sdM3.0 (error bar of half a subclass). The spectra of significantly metal-poor companions are dominated by strong $\mathrm{MgH}$ and $\mathrm{CaH}$ molecular absorptions. At the resolution of our data, spectra appear featureless redward of $800 \mathrm{~nm}$, except for the Ca II triplet, which remains detectable down to esdM3. The molecular features between 600 and $800 \mathrm{~nm}$ are quite sensitive to temperature changes, in contrast to the blue spectra.

Proper motion pairs have projected separations between $\sim 30$ and $\sim 57000$ AU. Very wide companions are also identified among the most metal-depleted stars. These orbital sizes are similar to those of solar-metallicity binaries and multiple stars. After correcting for the effect of increasing distance to the most metal-deficient stars in our survey, we determined that $13-15 \%$ of the low-metallicity G- to M-type stars harbor wide companions. This binary frequency is very similar to the binary fraction observed among stars of the solar vicinity and T Tauri stars of star-forming regions, suggesting that metallicity is not a key parameter in the stellar formation of wide double and multiple systems.

Acknowledgements. We thank R. Rebolo, M. Guerrero, E. Oblak, and J. Webb for their help with the observations. This publication makes use of data products from the Two Micron All Sky Survey (2MASS), which is a joint project of the University of Massachusetts and the Infrared Processing and Analysis Center/California Institute of Technology, funded by the National Aeronautics and Space Administration and the National Science Foundation. This research has made use of the SIMBAD database, operated at CDS, Strasbourg, France. It has also made use of the Digitized Sky Surveys produced at the Space Telescope Science Institute under U.S. Government grant
NAG W-2166. This work is partly financed by the Spanish projects AYA2003-053555 and Ramón y Cajal.

\section{References}

Abt, H. A., \& Willmarth, D. 1987, ApJ, 318, 786

Ake, T. B., \& Greenstein, J. L. 1980, ApJ, 240, 859

Allard, F., \& Hauschildt, P. H. 1995, ApJ, 445, 433

Allen, C., Poveda, A., \& Herrera, M. A. 2000, A\&A, 356, 529

Alonso, A., Arribas, S., \& Martínez-Roger, C. 1994, A\&AS, 107, 365

Alonso, A., Arribas, S., \& Martínez-Roger, C. 1996, A\&AS, 117, 227

Baraffe, I., Chabrier, G., Allard, F., \& Hauschildt, P. H. 1997, A\&A, 327,1054

Beers, T. C., Preston, G. W., \& Shectman, S. A. 1985, AJ, 90, 2089

Béjar, V. J.S., Zapatero Osorio, M. R., \& Rebolo, R. 2003, Astron. Nachr., submitted

Bessell, M. S., \& Brett, J. M. 1988, PASP, 100, 1134

Brandner, W., Alcalá, J. M., Kunkel, M., Moneti, A., \& Zinnecker, H. 1996, A\&A, 307, 121

Burgasser, A. J., Kirkpatrick, J. D., Cutri, R. M., et al. 2000, ApJ, 531, L57

Carney, B. W., Latham, D. W., Laird, J. B., \& Aguilar, L. A. 1994, AJ, 107,2240

Carpenter, J. M. 2001, AJ, 121, 2851

Cayrel de Strobel, G., Hauck, B., Francois, P., et al.1992, A\&AS, 95, 273

Cayrel de Strobel, G., Soubiran C., Friel, E. D., Ralite, N., \& Francois, P. 1997, A\&AS, 124, 299

Chabrier, G., \& Baraffe, I. 1997, A\&A, 327, 1039

Dahn, C. C., Liebert, J., Harris, H. C., \& Guetter, H. H. 1995, in The Bottom of the Main Sequence - and Beyond, ed. C. G. Tinney, ESO Astrophys. Symp. (Springer Verlag), 239

Giclas, H. L., Burnham, R., \& Thomas, N. G. 1971, in Lowell proper motion survey Northern Hemisphere, The G numbered stars. 8991 stars fainter than magnitude 8 with motions $>0$ '.26/year

Gizis, J. E. 1997, AJ, 113, 806

Gizis, J.E., \& Reid, I. N. 1997, PASP, 109, 1233

Gliese, W, \& Jahreiss, H. 1988, Ap\&SS, 142, 49

Goldberg, D., Mazeh, T., Latham, D. W., et al. 2002, AJ, 124, 1132

Greenstein, J. L. 1989, PASP, 101, 787

Hartwick, F. D. A., Cowley, A. P., \& Mould, J. R. 1984, ApJ, 286, 269

Laird, J. B., Carney, B. W., \& Latham, D. W. 1988, AJ, 95, 1843

Latham, D. W., Mazeh, T., Stefanik, R. P., et al. 1992, AJ, 104, 774

Latham, D. W., Stefanik, R. P., Torres, G., et al. 2002, AJ, 124, 1144

Lee, S.-G. 1984, AJ, 89, 702

Leggett, S. K. 1992, ApJS, 82, 351

Leggett, S. K., Golimowski, D. A., Fan, X., et al. 2002, ApJ, 564, 452

Lépine, S., Rich, R. M., \& Shara, M. M. 2003a, AJ, 125, 1598

Lépine, S., Shara, M. M., \& Rich, R. M. 2003b, ApJ, 585, L69

Luyten, W. J. 1970, Minneapolis: University of Minnesota

Luyten, W. J. 1979, Minneapolis: University of Minnesota, 2nd ed.

Martín, E. L., \& Rebolo, R. 1992, ASP Conf. Ser., 32, 336

Martín, E.L., Rebolo, R., \& Zapatero Osorio, M. R. 1995, in The Bottom of the Main Sequence and Beyond, ed. C. G. Tinney, ESO Astrophys. Symp. (Springer Verlag), 253

Monet, D. G., Dahn, C. C., Vrba, F. J., et al. 1992, AJ, 103, 638

Nakajima, T., Oppenheimer, B. R., Kulkarni, S. R., et al. 1995, Nature, 378, 463

Perryman, M. A. C., Lindegren, L., Kovalevsky, J., et al. 1997, A\&A, 323, L49

Poveda, A., Herrera, C. A., Cordero, G., \& Lavalley, G. 1994, Rev. Mex. Astron. Astrofis., 28, 43 
Rebolo, R., Zapatero Osorio, M. R., Madruga, S., et al. 1998, Science, 282, 1309

Reid, I. N., Hawley, S. L., \& Gizis, J. E. 1995, AJ, 110, 1838

Reid, I. N., Wyk, F. V., Marang, F., et al. 2001, MNRAS, 325, 931

Rieke, G. H., \& Lebofsky, M. J. 1985, ApJ, 288, 618

Ryan, S. G. 1989, AJ, 98, 1693

Ryan, S. G. 1992, AJ, 104, 1144

Ryan, S. G., \& Norris, J. E. 1991, AJ, 101, 1835

Salim, S., \& Gould, A. 2003, ApJ, 582, 1011

Saumon, D., Bergeron, P., Lunine, J. I., Hubbard, W. B., \& Burrows, A. 1994, ApJ, 424, 333
Schuster, W. J., \& Nissen, P.E. 1989, A\&A, 222, 69

Stauffer, J. R., \& Hartmann, L. W. 1986, ApJS, 61, 531

Tinney, C. G., Mould, J. R., \& Reid, I. N. 1993, AJ, 105, 1045

van Briesbroeck, G. 1961, AJ, 66, 528

Wegner, G. 1973, MNRAS, 163, 381

Weis, E. W. 1991, AJ, 101, 1882

Zakhozhaj, V. A., \& Shaparenko, E. F. 1996, Kinematika Fiz. Nebesn. Tel., 12, part No. 2, 20 